\title{
Multichannel matched filtering for spherical gravitational wave antennas
}

\author{
Carlos Filipe Da Silva Costa ${ }^{(1)}$, Stefano Foffa ${ }^{(2)}$ and Riccardo \\ Sturani $^{(3,4)}$
}

(1) Départment de Physique Nucléaire et Corpusculaire, Université de Genève

(2) Départment de Physique Théorique, Université de Genève, CH-1211 Geneva, Switzerland

(3) Istituto di Fisica, Università di Urbino, I-61029 Urbino, Italy

(4) INFN, Sez. Firenze, Sesto Fiorentino, I-50019 Italy

\author{
E-mail: Filipe.DaSilva@unige.ch, Stefano.Foffa@unige.ch, \\ Riccardo.Sturani@uniurb.it
}

\begin{abstract}
We study the performance of a multidimensional matched filter as a follow-up module of the coherent method recently developed by two of us for the detection of gravitational wave bursts by spherical resonant detectors. We have tested this strategy on the same set of injections used for the coherent method and found that the matched filter sensibly improves the determination of relevant parameters as the arrival time, amplitude, central frequency and arrival direction of the signal. The matched filter also improves the false alarm rate, reducing it roughly by a factor of 3 . The hierarchical structure of the whole analysis pipeline allows to obtain these results without a significant increase of the computation time.
\end{abstract}

PACS numbers: $04.80 . \mathrm{Nn}, 95.55 . \mathrm{Ym}$ 


\section{Introduction}

Whether the first direct gravitational wave (GW) detection will be made during the forthcoming joint LIGO-Virgo [1] run, or rather will be claimed by the next generation of interferometers, the intriguing era of gravitational wave astronomy is in any case expected to begin.

In this perspective, spherical resonant detectors, having the capability of locating on their own the gravitational wave source in the sky, may represent an important resource for this arising discipline. Contrarily to an interferometer, a sphere is a multichannel detector capable in principle to determine all the four parameters characterizing a GW (two angles for the arrival direction, and the amplitudes of the two polarizations); in order to deal with this distinguishing feature, several data analysis issues must be however tackled (see [2]- [12] for an incomplete but elucidating historical perspective).

In [13] and [14] a coherent burst search algorithm has been proposed, that fully exploits the multichannel capabilities of the sphere by transforming the detector's outputs into a five-dimensional time series corresponding to the spheroidal components of the gravitational wave. This time series is in turn processed by a coherent, waveletbased module [15] (henceforth CWS, as Coherent Waveburst for the Sphere) generating a list of candidate burst events endowed with all the relevant parameters (arrival time, arrival direction, amplitude, etc...) and is also able, to some extent, to reject nontransverse excitations (which are surely not due to GW's), thus reducing the false alarm rate by a factor of about 10 .

The reason of using wavelets rather than the traditional matched filter(MF)-ing technique is two-fold. MF-based searches are computationally expensive, as they require the exploration of a parameter space which is made extremely large by the presence of several channels (for example, the multichannel MF is direction dependent, differently from the traditional single channel one). Moreover wavelet-based searches, contrarily to a MF one, do not require any detailed prior knowledge of a signal, a very welcome feature for an unmodeled burst search such as the one developed in [13, 14].

On the other hand, as the use of the MF is known to give the optimal signal-to-noise ratio (SNR) [16], the wavelets flexibility has a cost in term of accuracy in the determination of the burst parameters.

To this respect, the present work can be considered as a natural evolution of [13, 14] in that it implements a multidimensional coherent MF as a follow-up of the method cited above. This hierarchical strategy is crucial in reducing the parameters space because for each trigger only a little region is explored around the values indicated by the CWS module, thus making the whole procedure reasonably fast.

The plan of the paper is the following: in section 2 the whole pipeline is briefly described, showing in particular how the MF module is interfaced to the previous part of the analysis chain; then in section 3 the improved method is tested against the same mock data sets (software injections of signals on a background endowed with both Gaussian and non-Gaussian noise, generated on the basis of a numerical simulation of 
the relevant components of the detector), thus showing the improvements obtained in the parameters estimation. Finally, section 4 contains our conclusions.

\section{Description of the analysis pipeline}

\subsection{From the transducers' outputs to the event candidates}

The first part of the pipeline, which is exhaustively discussed in [13] and [14, will be just briefly summarized here, leaving aside many technical details. The first step consists in converting the six transducers' outputs $I_{k}$ into five channels corresponding to the 5 quadrupolar modes $h_{N}$ of the GW tensor $h_{i j}$ :

$$
I_{k}(t) \longrightarrow h_{N}(t)
$$

with

$$
h_{i j}=\sum_{N=0,4} \mathcal{Y}_{m_{N}, i j}^{(2)} h_{N}, \quad Y_{m_{N}}^{(2)}=\sum_{i, j=x, y, z} \mathcal{Y}_{m_{N}, i j}^{(2)} n^{i} n^{j},
$$

where $m_{N}=0,1 c, 1 s, 2 c, 2 s$ labels the components of the real spherical harmonics $Y_{m_{N}}^{(2)}$ (defined and normalized as in [3]), $\mathcal{Y}_{m_{N}, i j}^{(2)}$ denotes the Cartesian component of the tensor spherical harmonics and $n^{i}$ is the versor of the arrival direction of the GW.

This conversion allows to extract the information about the GW from the detector's outputs (or, equivalently, to express its noisy content in term of its "GW-equivalent") and is possible once a mathematical model for the detector is available. In the case at hand, the model consists of a set of coupled oscillators describing the dynamics of the relevant sphere vibrational modes, of the transducers, and of the electrical circuit that are at the core of the readout devices (see [14] for details).

The next step is to combine the Fourier transforms of the five $h_{N}$ channels into a single channel $H$ :

$$
h_{N}(\omega) \longrightarrow H(\omega) \text {. }
$$

This process is performed by extracting from the dataset the spectral density matrix, defined from

$$
S_{N N^{\prime}}^{h}(\omega) \delta\left(\omega-\omega^{\prime}\right) \equiv<h_{N}(\omega) h_{N^{\prime}}^{*}\left(\omega^{\prime}\right)>,
$$

and then by building the following quantity:

$$
H(\omega) \equiv(h \cdot h)_{S} \equiv \sum_{N, N^{\prime}} h_{N}^{\dagger}(\omega) \cdot\left(S^{h}\right)_{N N^{\prime}}^{-1}(\omega) \cdot h_{N^{\prime}}(\omega) \text {. }
$$

This quantity is then fed into the CWS algorithm, which is a modified version of the Coherent Waveburst algorithm developed for LIGO ([17]-[20]), that has been adapted to our computational needs (a standard MacPro machine with two $3 \mathrm{GHz}$ processors) and to our detector configuration. This algorithm provides a list of triggers containing the various event features (central time of events, amplitude, arrival direction, central spectral frequency and spectral frequency spread). 
The trigger list is finally further refined through an independent determination of the arrival direction (the so-called determinant method), and a trigger is vetoed when the two reconstructed directions are not compatible. To summarize:

$$
H(\omega) \longrightarrow\{\text { triggers }\} \longrightarrow\{\text { event candidates }\}
$$

\subsection{Follow-up of the events via multidimensional matched filtering}

If the time dependence, arrival direction and polarization of the GW signal were known, that is if the expression for the five functions $h_{N}^{\text {sig }}(\omega)$ were available, the optimal detection strategy would be to perform a multidimensional matched filter. As explained in [6], the multichannel MF consists in analyzing the following scalar channel:

$$
H_{M F}(\omega) \equiv\left(h^{s i g} \cdot h\right)_{S},
$$

and is the strategy which gives the optimal SNR

$$
S N R=\frac{\int\left(h^{s i g} \cdot h\right)_{S} \mathrm{~d} \omega}{\sqrt{\int\left(h^{s i g} \cdot h^{s i g}\right)_{S} \mathrm{~d} \omega}}
$$

for the given signal $h^{\text {sig }}$. The integrals are done on both positive and negative frequencies. However, in a real experimental situation $h^{\text {sig }}$ is not known a priori and to employ efficiently a MF one should explore a huge number of points in a parameter space describing the temporal profile of the signal, as well as the arrival direction and polarization. Thus the use of a MF in such a context is computationally very demanding.

The CWS method exposed in the previous paragraph has been shown to find candidate events with a good efficiency/reliability ratio, without need to know the form of the signal. This allows to keep under control the computation time but of course it comes at a price for what concerns parameter estimation.

The improvement we are introducing in this work consists in performing a multidimensional MF only in the time segments where candidates events have been selected by the CWS, and to restrict the analysis to a small portion of parameter space centered around the parameters provided by the CWS triggers.

Specifically, we consider a two parameter family of sine-Gaussian shapes to describe the time profile of the signal

$$
h_{N}^{s i g}\left(t \mid t_{0}, \Delta, f_{0}, \theta, \phi\right)=h_{0} \mathrm{e}^{-\left(t-t_{0}\right)^{2} /\left(2 \Delta^{2}\right)} \sin \left[2 \pi f_{0}\left(t-t_{0}\right)\right] Y_{m_{N}}^{(2)}(\theta, \phi),
$$

impinging from the generic direction characterized by polar angles $\theta, \phi$. Since we have verified that the unmodeled search alone already gives a good estimation of the frequency spread $1 /(2 \pi \Delta)$ (an estimation which is not improved by the MF procedure), we kept it as fixed, in such a way that, for each candidate event we are basically exploring a small portion of the 4-dimensional parameter space $\left\{t_{0}, f_{0}, \theta, \phi\right\}$.

Such a space is explored through a maximum-seeking algorithm, which searches for the point characterized by the maximum SNR.

We maximized first on the arrival time, as the peak of the SNR is very sharp against this parameter, then on the central frequency and finally over the two angles of 


\begin{tabular}{|c|c|c|}
\hline$h_{r s s}\left(\mathrm{~Hz}^{-1 / 2}\right)$ & $\# \mathrm{GW}$ & \#non-GW \\
\hline $10^{-20}$ & 48 & 8 \\
\hline $7 \cdot 10^{-21}$ & 33 & 5 \\
\hline $5 \cdot 10^{-21}$ & 30 & 7 \\
\hline $3 \cdot 10^{-21}$ & 24 & 9 \\
\hline
\end{tabular}

Table 1. Detection efficiency for injected GW signals and non-GW disturbances. Adapted from [14].

the arrival direction together, keeping at each stage the other parameters fixed at the value indicated by the CWS triggers. This is a simplified choice of course, with respect to building a full parameter template bank, for instance, but it allows to obtain good results while keeping a low computational burden.

Once found, the maximum-SNR point in the parameter space defines the improved parameters of the candidate event, and if they happen to be too much different from the ones given by the unmodeled search, the event is discarded as spurious.

This means that the MF follow-up procedure, as well as improving the events' parameters, acts as a further veto against non-GW triggers, i.e. against signals which are not compatible with the transverse-traceless nature of GW's. Such false triggers can come from Gaussian noise or from non-Gaussian disturbancies. The CWS method alone is already optimized to reduce the latter's, and we will show that the application of the MF follow-up improves the performance with respect to the rejection of false triggers due to Gaussian noise.

\section{Results}

We have tested the MF method on the triggers obtained in [14], i.e. $50 \mathrm{GW}$-injections, ( 25 for each polarization) for each of the four $h_{r s s}$ amplitudes: $3,5,7,10 \times 10^{-21} \mathrm{~Hz}^{-1 / 2}$ and also on an equal number of non-GW injections (signals whose power has been distributed randomly among the five sphere channels) with the same amplitude. The injected signals are sine-Gaussian of the same type of the matching function given in (9), this allows us to assess the precision in parameter estimation irrespectively of a mismatch between the filter and the signal in the data. An eventual mismatch could of course worsen the estimations, but we do not investigate here on that.

For the sake of clarity, we have reported in table 1 the detection efficiency for both GW and non GW-injections obtained in [14]; these numbers, which show that the CWS alone already reduces by a factor of 5-10 the number of non-Gaussian false triggers, have not changed in the present analysis. 


\subsection{Efficiency vs. false alarm rate}

The GW detection efficiency alone is not a satisfactory measure of the goodness of the analysis method, as it must be completed by informations about the false alarm rate. A Receiver Operation Characteristic (ROC) curve has been constructed in order to find the set of analysis parameters (the most important being in our case the maximal allowed distance between the two direction reconstruction methods, $\left.\delta_{t h r}\right)$ that provides the optimal efficiency vs. false alarm performance.

In figure 1 we report the ROC curves both for the CWS unmodeled search and after the application of the MF, for different injection strengths. The false alarm rate is obtained from the analysis of simulated data containing Gaussian noise only. The figure shows that, at a given level of the efficiency, the MF method allows to reduce considerably the false alarm rate (by roughly a factor of 3 for $\delta_{t h r}=0.2$ radians, which the same working point chosen in [14]).

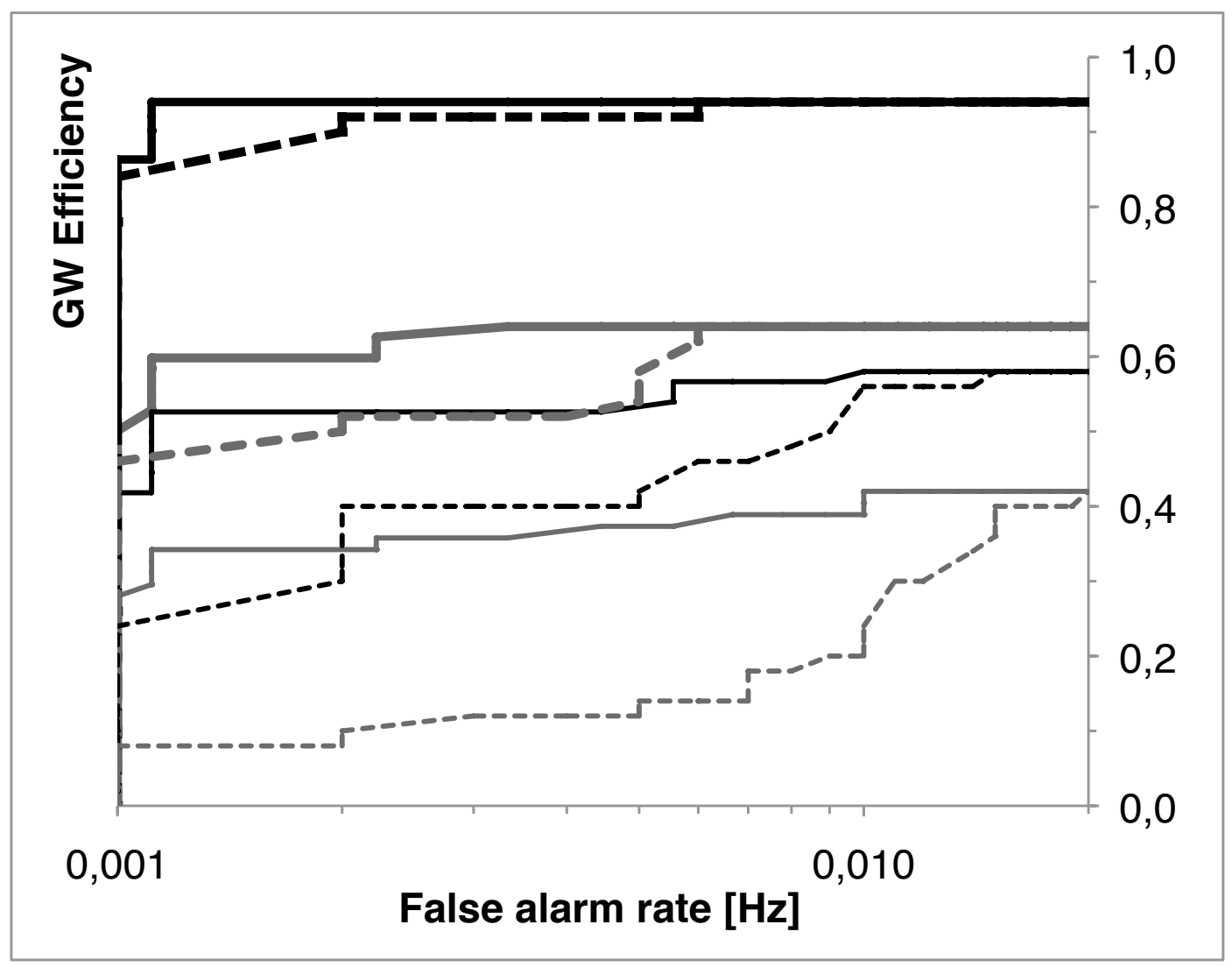

Figure 1. ROC curves (detection efficiency vs. false alarm rate) for the GWinjections, obtained by varying the maximum allowed distance between the direction reconstruction methods. Broken lines show the ROC obtained in 14] with the unmatched method, varying the distance between the CWS and the determinant method; solid lines show the results after the application of the matched filter and are obtained varying the distance between CWS and MF methods. Lines of increasing thickness correspond to injections sets of increasing amplitude, $h_{r s s}=$ $\{3,5,7,10\} \times 10^{-21} \mathrm{~Hz}^{-1 / 2}$. 


\subsection{Parameters estimation}

3.2.1. Arrival time The MF improves by an order of magnitude the arrival time estimation, as can be seen in figure 2, The results are summarized in table 2, where we plotted the average and the standard deviation of the quantities $t_{C W S}-t_{i n j}$ and $t_{M F}-t_{i n j}$.

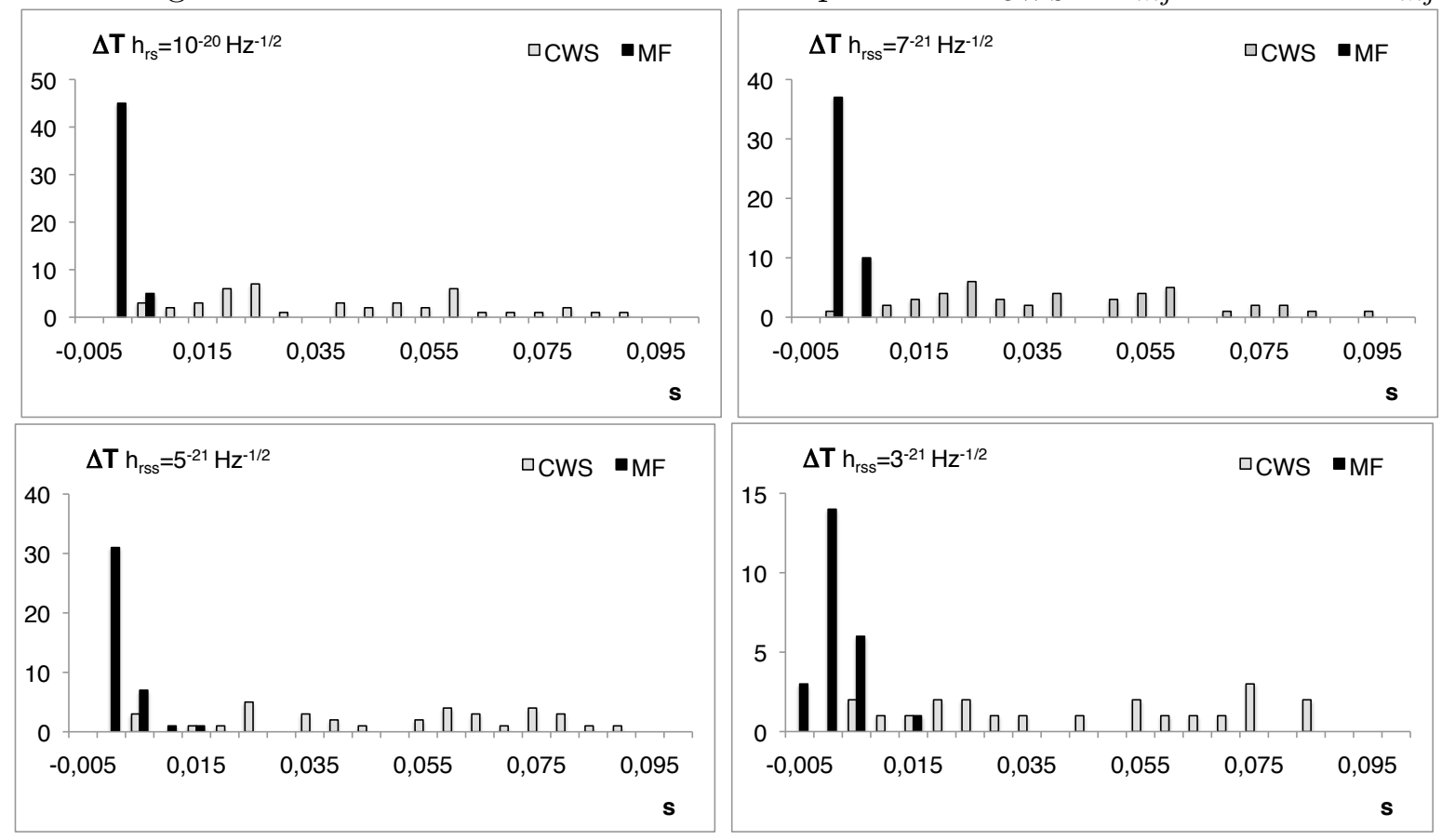

Figure 2. Histograms showing the arrival time reconstruction precision for the unmodeled (light) and matched-filtered (black) search, at different amplitudes.

\begin{tabular}{|c|c|c|c|c|}
\hline \multirow{2}{*}{$h_{r s s}\left(\mathrm{~Hz}^{-1 / 2}\right)$} & \multicolumn{2}{|c|}{$\Delta t_{U}(\mathrm{msec})$} & \multicolumn{2}{c|}{$\Delta t_{M F}(\mathrm{msec})$} \\
\cline { 2 - 5 } & $\mu$ & $\sigma$ & $\mu$ & $\sigma$ \\
\hline $10^{-20}$ & 43 & 28 & -1.2 & 2.1 \\
\hline $7 \cdot 10^{-21}$ & 43 & 25 & -0.48 & 2.9 \\
\hline $5 \cdot 10^{-21}$ & 52 & 28 & -0.24 & 3.4 \\
\hline $3 \cdot 10^{-21}$ & 48 & 29 & -0.40 & 4.7 \\
\hline
\end{tabular}

Table 2. Average $\mu$ and standard deviation $\sigma$ of the difference (in milliseconds) between the arrival time determination and the actual injected one, for the unmodeled search (U) and for the matched filter. The fact that $|\mu|<\sigma$ in the MF case means that our estimator is unbiased, and in such case the precision of the method is given by $\sigma$. 
3.2.2. Central frequency Also the estimation of $f_{0}$ benefits of an one order of magnitude improvement with the MF method, see table 3. More specifically, most of the improvement comes from the correction of a few very imprecise estimations given by the CWS, as can be seen in the histograms of figure 3 .
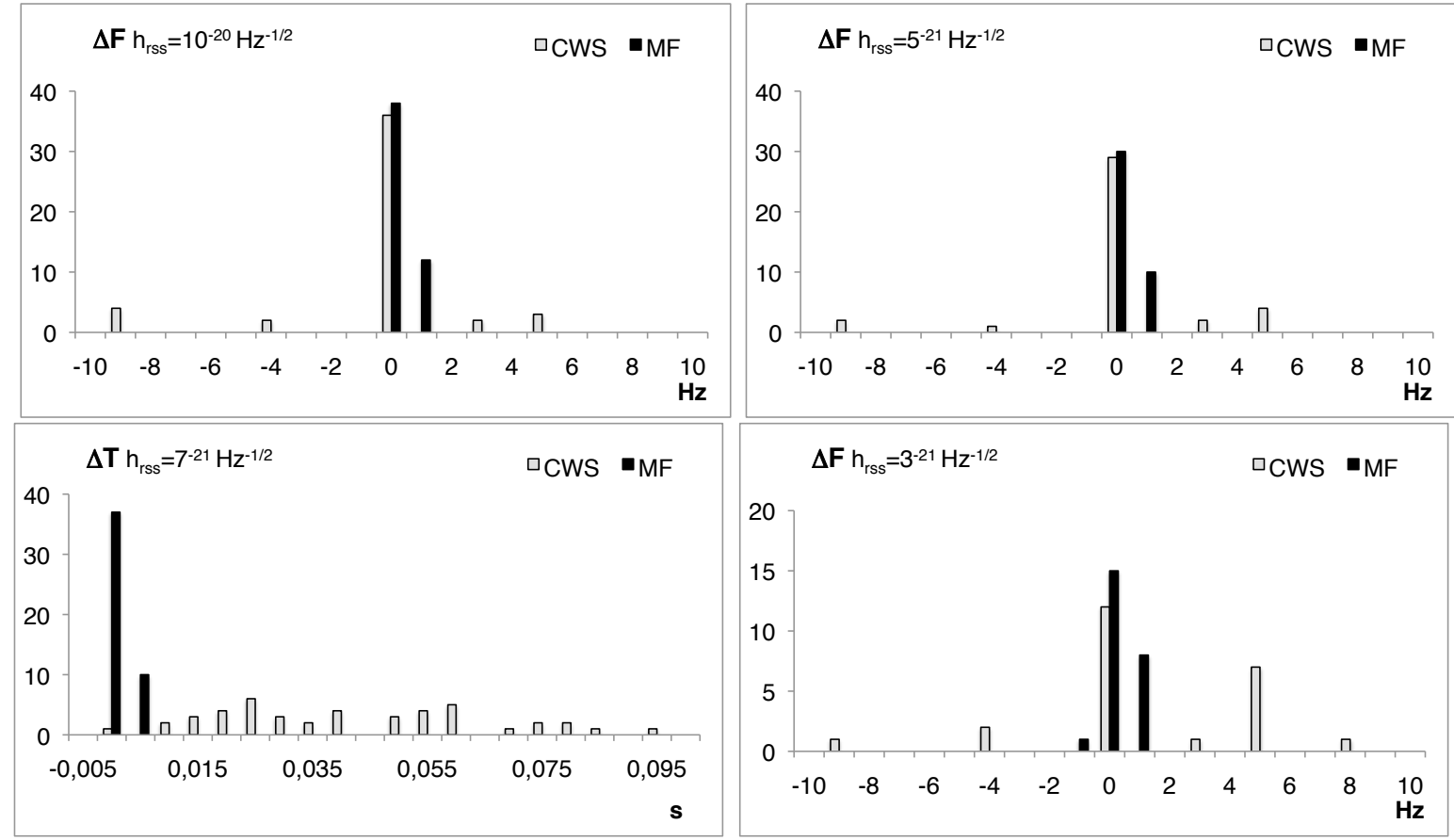

Figure 3. The four histograms show the distribution of the quantities $f_{0}^{C W S}-f_{0}^{i n j}$ (light) and $f_{0}^{M F}-f_{0}^{i n j}$ at different injected amplitudes.

\begin{tabular}{|c|c|c|c|c|}
\hline \multirow{2}{*}{$h_{r s s}\left(\mathrm{~Hz}^{-1 / 2}\right)$} & \multicolumn{2}{|c|}{$\Delta f_{0}^{U}(\mathrm{~Hz})$} & \multicolumn{2}{|c|}{$\Delta f_{0}^{M F}(\mathrm{~Hz})$} \\
\cline { 2 - 5 } & $\mu$ & $\sigma$ & $\mu$ & $\sigma$ \\
\hline $10^{-20}$ & 0.28 & 4.6 & 0.08 & 0.28 \\
\hline $7 \cdot 10^{-21}$ & 0.60 & 4.7 & 0.08 & 0.32 \\
\hline $5 \cdot 10^{-21}$ & 0.70 & 4.1 & 0.10 & 0.27 \\
\hline $3 \cdot 10^{-21}$ & 0.97 & 3.6 & 0.06 & 0.54 \\
\hline
\end{tabular}

Table 3. Average $\mu$ and standard deviation $\sigma$ of $\Delta f_{0}$ (in $\mathrm{Hz}$ ) for the unmodeled search (U) and for the matched filter. The fact that $|\mu|<\sigma$ means that our estimator is unbiased, and in such case the precision of the method is given by $\sigma$. 
3.2.3. Arrival direction In fig. 4 the direction reconstruction of the unmodeled and of the MF search are plotted. In this case the improvement, although clearly visible, is not as spectacular as in the previous cases, and appears to be more marked for $\phi$ than for $\theta$; this last feature might depend on the chosen injection direction, and further study is necessary to understand the behavior for generic arrival directions. Quantitative results for the angular distance $\delta s$ are summarized in tab. 4 .

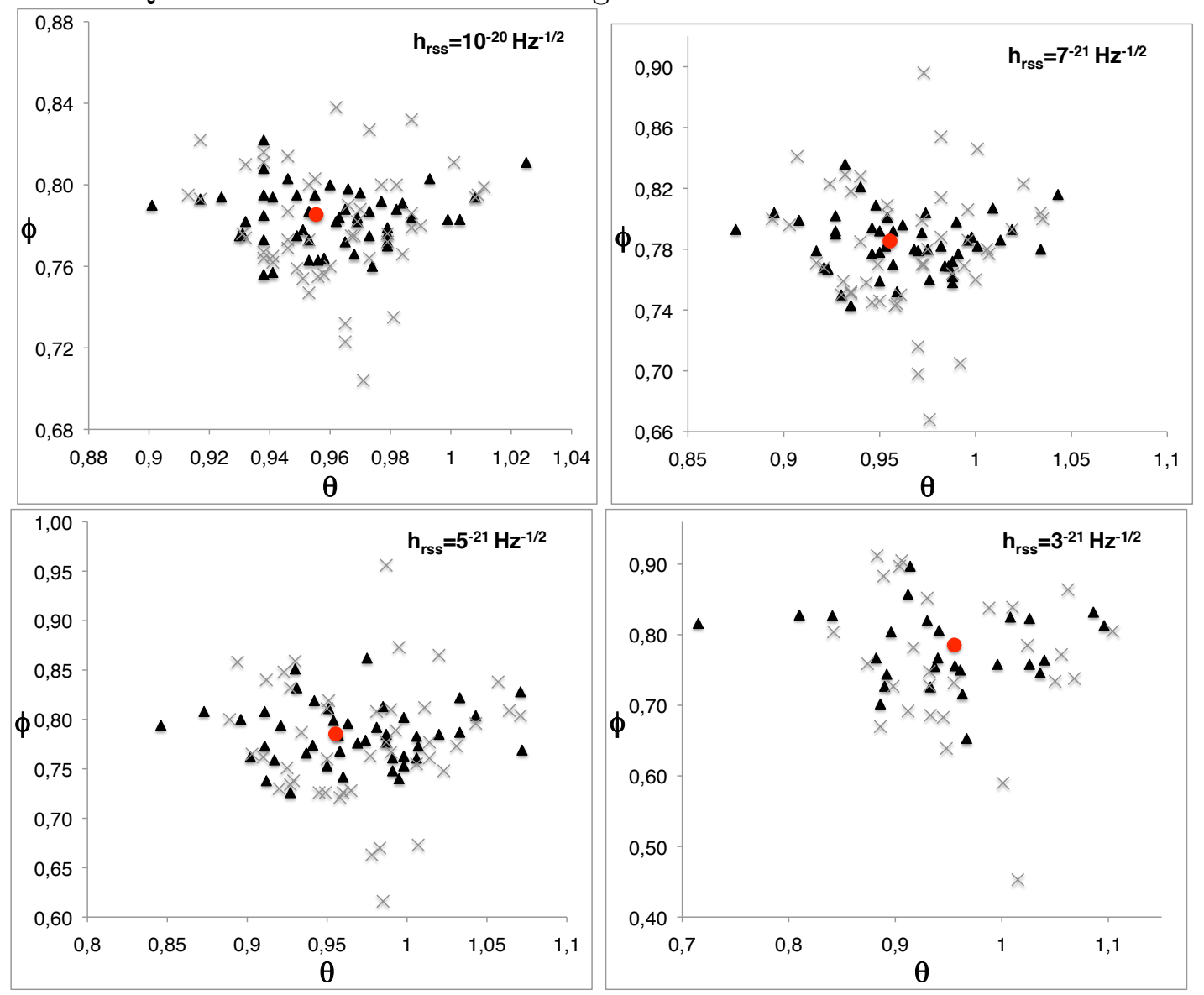

Figure 4. Arrival direction reconstruction by the unmodeled search (crosses) and by the MF (triangles) for the four given signal amplitudes. The circle represents the injection direction.

\begin{tabular}{|c|c|c|}
\hline$h_{r s s}\left(\mathrm{~Hz}^{-1 / 2}\right)$ & $\delta s_{U}$ & $\delta s_{M F}$ \\
\hline $10^{-20}$ & 0.030 & 0.024 \\
\hline $7 \cdot 10^{-21}$ & 0.044 & 0.034 \\
\hline $5 \cdot 10^{-21}$ & 0.58 & 0.050 \\
\hline $3 \cdot 10^{-21}$ & 0.092 & 0.083 \\
\hline
\end{tabular}

Table 4. Average angular distance (in radians) between the direction determination and the actual injection direction, for the unmodeled search (U) and for the matched filter. Differently from the previous cases, the angular distance is clearly a biased parameter (because it is not distributed around zero, by definition), so the accuracy of the estimation is well described by the average. 


\begin{tabular}{|c|c|c|}
\hline \multirow{2}{*}{$h_{r s s}\left(\mathrm{~Hz}^{-1 / 2}\right)$} & \multicolumn{2}{|c|}{$\Delta h_{0}^{M F} / h_{0}$} \\
\cline { 2 - 3 } & $\mu$ & $\sigma$ \\
\hline $10^{-20}$ & $2.9 \cdot 10^{-3}$ & 0.057 \\
\hline $7 \cdot 10^{-21}$ & $3.5 \cdot 10^{-3}$ & 0.066 \\
\hline $5 \cdot 10^{-21}$ & $2.0 \cdot 10^{-3}$ & 0.061 \\
\hline $3 \cdot 10^{-21}$ & $2.5 \cdot 10^{-3}$ & 0.086 \\
\hline
\end{tabular}

Table 5. Average $\mu$ and standard deviation $\sigma$ of $\left(h_{0}^{M F}-h_{0}^{i n j}\right) / h_{0}^{i n j}$. The fact that $|\mu|<\sigma$ means that our estimator is unbiased, and in such case the precision of the method is given by $\sigma$.

3.2.4. Amplitude The CWS method have not been explicitly calibrated to measure the amplitude of the signal. However, it is still interesting to see that with the use of the Matched Filtering the relative precision in the determination is a few percents, as reported in in table 5 .

\section{Conclusions}

Matched filtering techniques are widely used in gravitational wave analysis. However they can have a high computational cost, because of the possibly large volume of parameter space required by a comprehensive search. The case of spherical detector is even worse as its multimode feature makes it highly sensitive to the arrival direction, adding the source position in the sky as further parameter.

Here we have shown how, in the case of burst searches, combining an unmodeled search with a matched one can exploit the goodies of both while taming the baddies of both. The unmodeled makes no assumption on the type of signal, but gives a non-optimal estimation of the parameters of the burst events triggered. On the other hand the matched filtered search is optimal once the shape of the signal is known. Here we use the output triggers of the unmodeled search to massively restrict the search in the parameter of the best matching template; more specifically we used a 4-dimensional parameter space (arrival time, central frequency and source angles in the sky) to present an example of matched filtering search. Our search is very much simplified with respect to a general one as the parameter space is not fully explored.

To make a comprehensive analysis one would need template banks and systematic scanning of the parameter space, but even with this primitive attempt we have shown how the first estimate made by the unmodeled search can be improved by a very light matched filter search.

\section{Acknowledgments}

It is a pleasure to thank M. Maggiore and M. Pohl for useful discussions and encouragement. The work of FS is supported by the Fond National Suisse. 


\section{References}

[1] www.ligo.caltech.edu, www.virgo.infn.it,

[2] R. V. Wagoner and H. J. Paik (1977). In Experimental Gravitation, Proceedings of the Pavia International Symposium, Accademia Nazionale dei Lincei, Roma.

[3] C. Z. Zhou and P. F. Michelson, Phys. Rev. D 51 (1995) 2517.

[4] E. Coccia, J. A. Lobo and J. A. Ortega, Phys. Rev. D 52, 3735 (1995).

[5] J. A. Lobo and M. A. Serrano, Europhys. Lett. 35, 253 (1996).

[6] T. R. Stevenson, Phys. Rev. D 56 (1997) 564.

[7] S. M. Merkowitz and W. W. Johnson, Phys. Rev. D 56 (1997) 7513 arXiv:gr-qc/9706062.

[8] S. M. Merkowitz, Phys. Rev. D 58 (1998) 062002 arXiv:gr-qc/9712079.

[9] J. A. Lobo, Mon. Not. Roy. Astron. Soc. 316 (2000) 173 arXiv:gr-qc/0006109].

[10] M. A. Gasparini and F. Dubath, Phys. Rev. D74 (2006), 122003.

[11] C. A. Costa and O. D. de Aguiar, arXiv:gr-qc/0606112.

[12] L. Gottardi, Phys. Rev. D 75 (2007), 022002.

[13] S. Foffa and R. Sturani, Class. Quant. Grav. 25 (2008) 184036 arXiv:0805.0718 [gr-qc]].

[14] S. Foffa and R. Sturani, Class. Quant. Grav. 26 (2009) 105013 arXiv:0812.4149 [gr-qc]].

[15] Mallat S. "A wavelet tour in signal processing", Academic Press, 1998, chap. VII.

[16] M. Maggiore, "Gravitational Waves. Vol. 1: Theory and Experiments", Oxford University Press, October 2007. 572p.

[17] S. Klimenko, I. Yakushi, M. Rakhmanov and G. Mitselmakher, 2004 Class.Quant.Grav. 21 S1685.

[18] S. Klimenko and G. Mitselmakher, 2004 Class.Quant.Grav. 21 S1819.

[19] S. Klimenko, S. Mohanty, M. Rakhmanov and G. Mitselmakher, Phys. Rev. D 72 (2005) 122002 arXiv:gr-qc/0508068.

[20] S. Klimenko, I. Yakushin, A. Mercer and G. Mitselmakher, arXiv:0802.3232 [gr-qc], to appear in the proceedings of GR18: 18th International Conference on General Relativity and Gravitation 7th Edoardo Amaldi Conference on Gravitational Waves Amaldi7), Sydney, Australia, 8-13 Jul 2007. 